\title{
Species diversity, biological spectrum and phenological behavior of vegetation of a Muslim sacred grove in Southwest Bengal, India
}

\author{
UDAY KUMAR SEN", RAM KUMAR BHAKAT \\ Department of Botany and Forestry, Ecology and Taxonomy Laboratory, Vidyasagar University. Vidyasagar University Rd, Midnapore 721102, \\ West Bengal, India. Tel.: +91-900-2524806, `email: uudaysen@gmail.com
}

Manuscript received: 3 October 2021. Revision accepted: 22 November 2021.

\begin{abstract}
Sen UK, Bhakat RK. 2021. Species diversity, biological spectrum and phenological behavior of vegetation of a Muslim sacred grove in Southwest Bengal, India. Nusantara Bioscience 13: 219-231. Sacred groves are an age-old and worldwide phenomenon. They are traditionally made up of forest or surrounding forest zones that local people safeguard based on their spiritual relationship with the deities or ancestral spirits believed to reside in them. Social practices play a crucial role in conserving the environment and biodiversity. This research aimed to examine the current flora of a sacred grove's plant resources, biological spectrum, leaf size spectra, conservation status, and management of the Faringdanga Pir Babar Asthana in the Paschim Medinipur District of West Bengal in India. The floristic element of the study area revealed that the vegetation was diverse and composed of 162 species belonging to 148 genera over 51 families under 26 orders according to the Angiosperm Phylogeny Group (APG) IV classification. Because the study area is a sacred grove, it is relatively undisturbed, and traditional beliefs ensure that germplasm in the grove is protected. The current study could serve as a model for phytoclimatic research in India's sacred forests.
\end{abstract}

Keywords: APG IV, biodiversity conservation, biological spectrum, conserved community area, leaf spectra, life form.

\section{INTRODUCTION}

Sacred groves of sacred forests are natural forest patches preserved by local people's religious beliefs, and they serve as a treasure trove of animal and plant species (Parthasarathy and Babu 2021). Sacred groves are traditional resource systems associated with a culture-based conservation paradigm (Hailemariam 2019; Sen and Bhakat 2021b). Furthermore, sacred groves may play a role in conserving biological diversity, ecological functions, ecosystem services, and cultural diversity (Blicharska et al. 2013). A typical sacred grove can range from clumps of a few trees to a few hectares and is usually found near human settlements. Physically, it's a tiny or massive area of forested ground with a mosque inside, but culturally, it's linked to ancestral spirits, myths, rituals, and taboos (Singh et al. 2021). These crucial customary duties have long protected the integrity of sacred groves and appear to play an important role in biodiversity conservation (Aniah and Yelfaanibe 2016). Sacred groves are also known as natural museums of tall or massive trees, medicinal plant repositories, treasure houses of endangered species, urban recreation centers, watershed regulators, a veritable garden for botanists, gene banks of economically important species, environmental laboratories, and ideal places for nature lovers (Parthasarathy and Babu 2021). Biological Diversity Act 2002 has provided legal provisions for conserving significant areas from a biodiversity point of view, as are critical cultural spaces such as sacred groves/trees and sites or other large community conserved areas (NBA 2021). In this regard, the Government of West Bengal has declared Chilkigarh Kanak Durga Sacred Grove, a 55.9 acres remnant forest patch in the Jhargram
District of West Bengal, as a Biodiversity Heritage Site (NBA 2021; WBBB 2021).

Climate determines the types of plants that can thrive in each habitat, and physiognomy refers to the overall appearance of vegetation. It describes the general structure, shape, and life forms of the species that make up the vegetation; physiognomy has been used to classify vegetation types (Hunter et al. 2021). The individual species in a community can be categorized into numerous life forms based on their physiognomy appearance and growth performance. The vegetation's life form is determined by its genetic pool and tolerance for climatic fluctuation. Biological spectra are also essential physiognomic features employed extensively in vegetation study. The spectrum of life forms is thought to be markers of micro and macroclimate (Khan et al. 2018). Leaf size groups, likewise, have been discovered to be highly effective for connections. Knowing leaf size may aid in studying plant physiological processes and communities (Nicotra et al. 2011). The literature on Paschim Medinipur district plant ecology reveals relatively little research on vegetation analyses, life forms, and leaf size spectrum. The method of Raunkiaer (1934) explains and aids in comprehending vegetative flora and structure in connection to current eco-biological conditions. It also represents the impact of contemporary biotic factors on the overall structure and composition of the vegetation, such as overgrazing, overharvesting, and deforestation. In addition, it has various effects on the economic worth of plants. Therefore, these methods can create a sustainable and profitable management plan for plant resource harvesting.

In light of the applications mentioned above for Raunkiaer's concepts, the present effort was started with the 
goal of (1) determining variation in life form and leaf size spectra in different plant communities from various climatic zones; (2) Based on the species list, analyze the Raunkiaerian spectra, and (3) elucidate the relationship between vegetation and religious gradient in the study area. Furthermore, the impact of anthropogenic activities and environmental factors on the observed patterns is also discussed.

\section{MATERIALS AND METHODS}

\section{Location of the study site and physiography}

Consisting of mixed vegetation of deciduous, semideciduous, and evergreen species, the present sacred grove (latitude $22^{\circ} 26^{\prime} 45.56^{\prime \prime} \mathrm{N}-22^{\circ} 26^{\prime} 40.92^{\prime \prime} \mathrm{N}$ and longitude $87^{\circ} 19^{\prime} 02.52^{\prime \prime} \mathrm{E}-87^{\circ} 19^{\prime} 01.15^{\prime \prime} \mathrm{E}$; average altitude $52 \mathrm{~m}$ asl), popularly known as Faringdanga Pir Babar Asthana (named after its adjacent locality and presiding saints Hazarat Golap Shah and Hazarat Jamaluddin Shah) is situated at the northern part of the Paschim Medinipur District, West Bengal, India. The grove is positioned neighboring Abas Khasjangal Cantonment under the Midnapore Sadar block in the Paschim Medinipur district along the Jharkhand state's border area. It is bordered on the north by the Purulia and Bankura districts and on the west by the district of Jhargram. It has widespread borders with the state of Orissa on the south and the west by the Jharkhand state (Figure 1). The climate is subtropical and humid. In the hot and dry days of May and June, temperatures can reach $46^{\circ} \mathrm{C}$, but on the chilly nights of December and January, it can fall to $8^{\circ} \mathrm{C}$.

The district of Paschim Medinipur under the Chota Nagpur Plateau covers an area of $6,308 \mathrm{~km}^{2}$. It has a population of $5,913,457$ per the 2011 census, with $87.78 \%$ of the total population being rural and only $12.22 \%$ being urban. The 2011 census showed that $19.07 \%$ and $14.88 \%$ of the population belonged to the scheduled castes and tribes, respectively (Anon 2011).

This 6-hectare grove represents several chunks of a 300500 year isolated forest patch in its near-climax stage and is bounded by abandoned fields. The Sufi saints Hazarat Golap Shah and Hazarat Jamaluddin Shah (believed to be offsprings of Hazarat Mohammed) stayed and practiced religious rituals in the mosque's area; after his death, his mortal body was buried in the said mosque. During the Urus-pak festival (on $22 / 23^{\text {rd }}$ February and $17 / 18^{\text {th }}$ July, devotees pray to the saints and seek blessing and well-being). Presently, the entire grove is maintained and managed by the descendants of the saints. The whole geographical area and its biotic and abiotic elements are revered. There is a social prohibition on cutting any plant specimen and even lifting leaf litter from the grove is a taboo. To celebrate the worship, devotees collect money and other utensils from everywhere throughout the neighboring areas of the region. The local communities maintain the sanctity of the whole area.
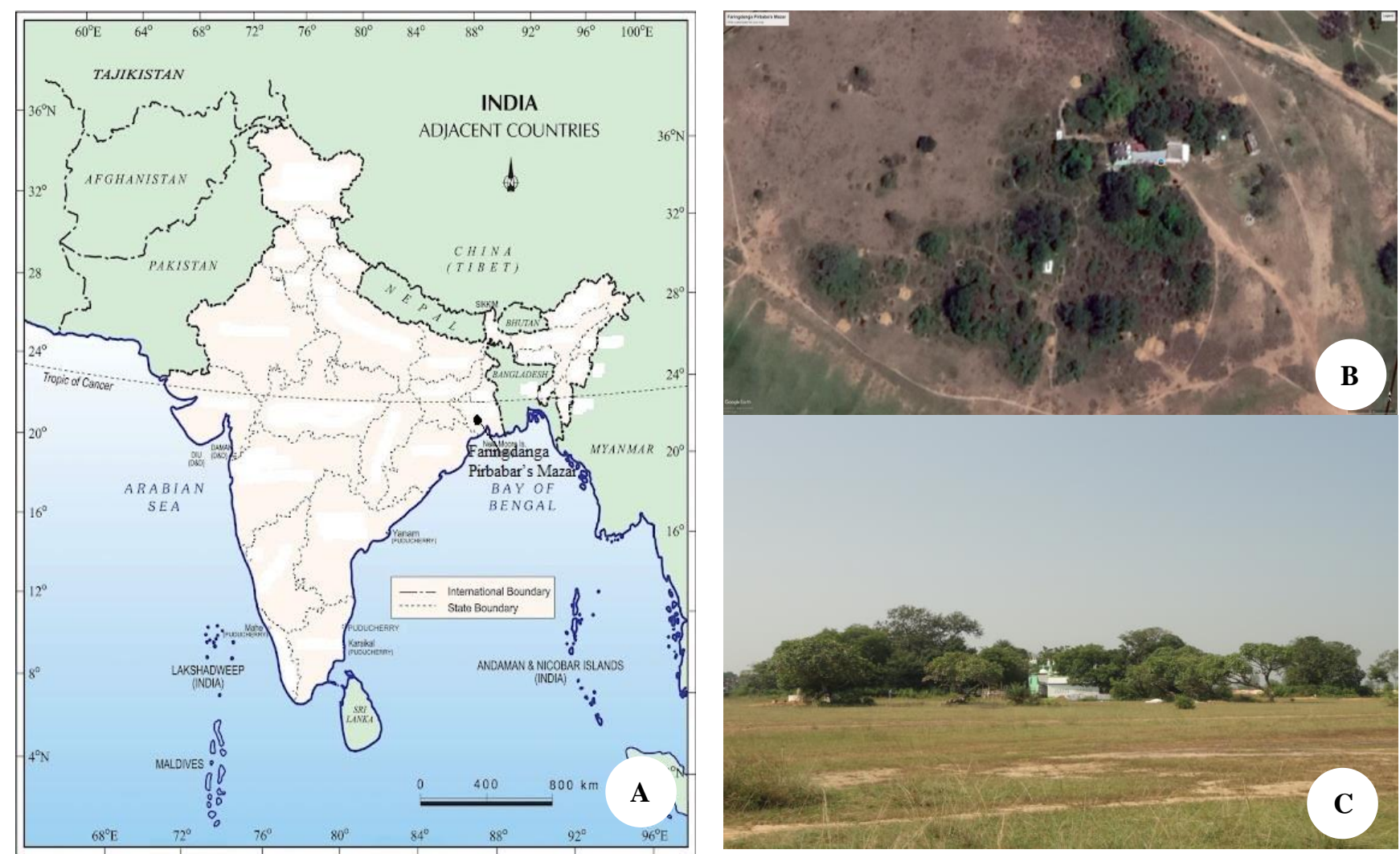

Figure 1. Location of the study area in Paschim Medinipur District, West Bengal, India: A. Faringdanga Pirbabar Mazar in West Bengal of India; B. Google Earth image of the study area; C. Faringdanga Pirbabar Mazar sacred grove in West Bengal of India 


\section{Field survey and data collection}

Before the field survey, thorough permission was taken from Faringdanga Pir Babar Asthana sacred grove management to study and collect plant samples. The study area was thoroughly investigated during the year 20122018 in different seasons. It presented a prospect of composting plant compilation and field interpretation during the entire flowering and fruiting of the maximum species quantity. Phytosociological data were collected by laying $20 \mathrm{~m} \times 20 \mathrm{~m}$ quadrates for tree species, $5 \mathrm{~m} \times 5 \mathrm{~m}$ for shrubs and lianas, and $1 \mathrm{~m} \times 1 \mathrm{~m}$ for herbs and grasses. Based on "spot identification," a brief floristic study was conducted. The specimens were processed, poisoned, mounted on herbarium sheets, and preserved in Vidyasagar University's Herbarium. Photographs of some common, locally uncommon, endemic, and valuable plants in the sacred grove were taken. The herbarium sheets were checked with annotated materials available at Vidyasagar University's Herbarium Section and the Botanical Survey of India. Various related catalogs, regional flora (Prain 1903), monographs, revision works, and other literature (Paria 2005; Anon 2010) have been consulted for identification purposes. The plant's scientific name was checked with the World Checklist of Vascular Plants (WCVP 2021) website, and only the accepted names were used.

\section{Analysis of vegetation}

According to the Angiosperm Phylogeny Group (APG) IV classification, the floristic list was taxonomically arranged by clade, order, and family according to Angiosperm Phylogeny Group (APG) IV classification (Chase et al. 2016). For each species, several voucher specimens, habit, life span, nativity, flowering and fruiting time, Raunkiaer's life form with sub-type, leaf spectra, the lamina shape, IUCN status (IUCN 2021), and plant growing seasons were inferred (Table 1). The resulting biological spectrum was then compared to Raunkiaer's standard spectrum to identify the grove's phytoclimate (Raunkiaer 1934; Mueller-Dombois and Ellenberg 1974). Leaf size information was employed to assess plants physiological systems and identify plant associations. The Raunkiaer (1934) figure was used to estimate the number of leaves in the field. Finally, a list of invasive alien species was prepared according to Reddy et al. (2008).

\section{RESULTS AND DISCUSSIONS}

\section{Taxonomic composition}

A total of 162 taxa belonging to 148 genera distributed in 51 families from 26 orders were recorded from the sacred grove. The top two clades were Asterids and Rosids. More than $79 \%$ of the flora was represented by Eudicot and Core Eudicot orders. The most represented orders ( $\geq 10$ species) were from Poales 21 (12.96\%), Fabales 18 (11.11\%), Lamiales 14 (8.64\%), Gentianales 13 (8.02\%), Malpighiales $13(8.02 \%)$ and Asterales 12 (7.41\%) (Table 1, Figure 2). Gastauer and Neto (2017) highlighted a similar distribution from Brazil. Gnanasekaran et al. (2012) conducted similar research on angiosperms in a sacred grove in the Cuddalore area of Tamil Nadu, India. Pérez-Luque et al. (2014) and Sen and Bhakat (2021a) have illustrated similar types of order contributions.

The eleven best-represented families (with $\geq 5$ species) were Fabaceae 18 (11.11\%), Poaceae 14 (8.64\%), Asteraceae 12 (7.41\%), Euphorbiaceae 9 (5.56\%), Malvaceae 9 (5.56\%), Apocynaceae 7 (4.32\%), Cyperaceae 7 (4.32\%), Lamiaceae 7 (4.32\%), Rubiaceae 6 (3.70\%), Acanthaceae $5(3.09 \%)$ and Menispermaceae $5(3.09 \%)$ (Table 1, Figure 3). Whereas three families contained 4 $(2.47 \%)$, four families contained $3(1.85 \%)$, and six families comprised $2(1.23 \%)$ species each. The other 27 families had only a single species (Table 1 ).

In India, several studies (Ghildiyal et al. 2016; Sen and Bhakat 2019, 2020) have discovered the same type of dominant families in sacred groves, and many other authors have demonstrated hegemony in the global context (Badshah et al. 2016; Khan et al. 2018; Farooq et al. 2019; Sherwani 2019; Asif et al. 2020; Haq et al. 2021). Moreover, they consistently backed our findings, with the Fabaceae, Poaceae, and Asteraceae families emerging as the most common study region.

The most represented genera were Cyperus and Solanum, each containing 4 and 3 species, respectively. Nine genera, e.g., Chrysopogon, Cleome, Croton, Dioscorea, Euphorbia, Senna, Sida, Spermacoce, and Ziziphus, hold 2 species each. Another 137 species contain a single genus respectively (Table 1).

\section{Species diversity in different growth forms}

The present floristic study of the sacred grove showed that they harbored a total of 162 plant species [130 dicots $(80.25 \%)$ and 32 monocots $(19.75 \%)]$ belonging to 148 genera [121 dicots $(81.76 \%)$ and 27 monocots (18.24\%)] under 51 families [41 dicots $(80.39 \%)$ and 10 monocots $(19.61 \%)$ ] in 26 orders [19 dicots $(73.08 \%)$ and 7 monocots (26.92\%)]. Among these, 79 (48.76\%) reported species were herbs. Other highly reported species were 30 shrubs (18.52\%), 23 trees $(14.20 \%)$, and 30 climbers (18.52\%), respectively. Amongst the total 130 dicots $(80.25 \%)$ and 32 monocots (19.75\%), herbs, shrubs, trees, and climbers represented 54, 29, 21, 26, and 25, 1, 2, 4 species, respectively, representing $33.33 \%, 17.90 \%, 12.96 \%$, $16.05 \%$ and $15.43 \%, 0.62 \%, 1.23 \%, 2.47 \%$ of the total species (Table $1 \& 2$, Figure 4).

\section{Life span and nativity}

In the sacred grove, 68 annual plants (39.63\%) go through their life cycle in one growing season, and 94 perennial plants $(59.91 \%)$ can survive the most unfavorable conditions and stay alive for more than two years (Table 1). Of the total recorded 162 species, 104 species were native, while 58 species were aliens, most of which thrive in the grove's disturbed habitats. Alien plant species were frequently able to colonize disturbed sites more quickly than native species, independent of their life history strategy, because they tended to be more plastic than native plants and, in some fitness components, superior to natives (Davidson et al. 2011). 
Table 1. List of angiosperm taxa of Faringdanga Pir Babar Asthana sacred grove, West Bengal, India, according to the APG IV classification system

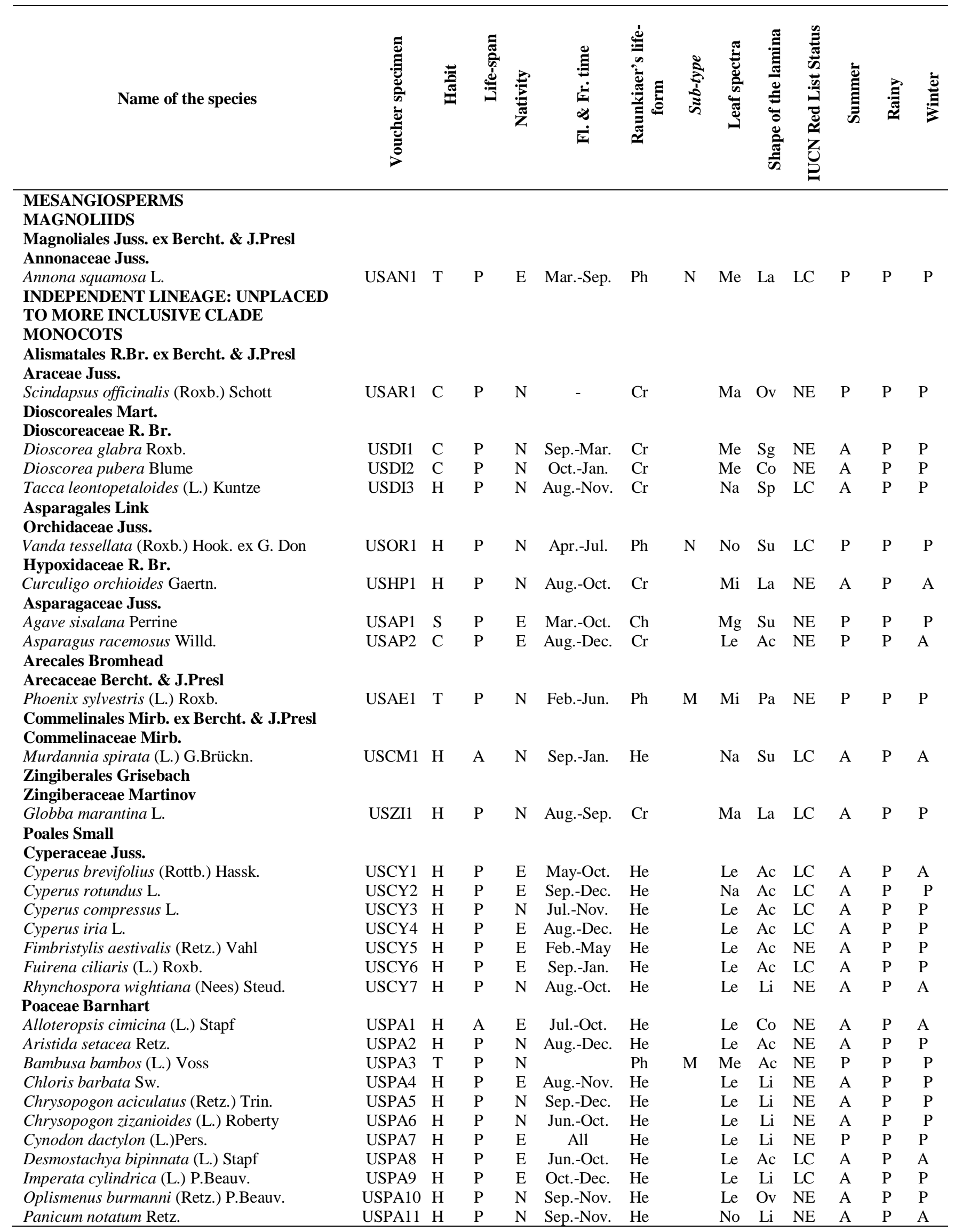




\begin{tabular}{|c|c|c|c|c|c|c|c|c|c|c|c|c|c|}
\hline Perotis indica (L.) Kuntze & USPA12 & & $\mathrm{P}$ & $\mathrm{N}$ & Jul.-Nov. & $\mathrm{He}$ & & $\mathrm{Le}$ & Ac & $\mathrm{NE}$ & $\mathrm{A}$ & $\mathrm{P}$ & A \\
\hline Setaria parviflora (Poir.) Kerguélen & USPA13 & $\mathrm{H}$ & $\mathrm{P}$ & $\mathrm{N}$ & Aug.-Nov. & $\mathrm{He}$ & & $\mathrm{Le}$ & $\mathrm{Li}$ & $\mathrm{LC}$ & A & $\mathrm{P}$ & A \\
\hline Tragus mongolorum Ohwi & USPA14 & $\mathrm{H}$ & $\mathrm{P}$ & $\mathrm{N}$ & Aug.-Oct. & $\mathrm{He}$ & & $\mathrm{Le}$ & Ac & $\mathrm{NE}$ & A & $\mathrm{P}$ & \\
\hline \multicolumn{14}{|l|}{ EUDICOTS } \\
\hline \multicolumn{14}{|c|}{$\begin{array}{l}\text { Ranunculales Juss. ex Bercht. \& J.Presl } \\
\text { Papaveraceae Juss. }\end{array}$} \\
\hline $\begin{array}{l}\text { Argemone mexicana L. } \\
\text { Menispermaceae Juss. }\end{array}$ & USPP1 & $\mathrm{H}$ & A & $\mathrm{E}$ & Dec.-Apr. & Th & & Ma & $\mathrm{Sp}$ & $\mathrm{NE}$ & $\mathrm{P}$ & A & \\
\hline Cissampelos pareira $\mathrm{L}$. & USMN1 & $\mathrm{C}$ & $\mathrm{P}$ & $\mathrm{N}$ & Jul.-Jan. & $\mathrm{Ph}$ & $\mathrm{N}$ & $\mathrm{Me}$ & Co & $\mathrm{NE}$ & $\mathrm{P}$ & $\mathrm{P}$ & \\
\hline Cocculus hirsutus (L.) W.Theob. & USMN2 & $\mathrm{C}$ & $\mathrm{P}$ & $\mathrm{N}$ & Aug.-Nov. & $\mathrm{Ph}$ & $\mathrm{N}$ & $\mathrm{Me}$ & Co & NE & $\mathrm{P}$ & $\mathrm{P}$ & \\
\hline Stephania japonica (Thunb.) Miers & USMN3 & $\mathrm{C}$ & $\mathrm{P}$ & $\mathrm{N}$ & Jul.-Dec. & $\mathrm{Ph}$ & $\mathrm{N}$ & $\mathrm{Me}$ & Or & $\mathrm{NE}$ & $\mathrm{P}$ & $\mathrm{P}$ & \\
\hline Tiliacora acuminata (Lam.) Miers & USMN4 & $\mathrm{C}$ & $\mathrm{P}$ & $\mathrm{N}$ & Nov.-May & $\mathrm{Ph}$ & $\mathrm{N}$ & $\mathrm{Me}$ & Ov & $\mathrm{NE}$ & $\mathrm{P}$ & $\mathrm{P}$ & \\
\hline Tinospora sinensis (Lour.) Merr. & USMN5 & $\mathrm{C}$ & $\mathrm{P}$ & $\mathrm{N}$ & Feb.-Jun. & $\mathrm{Ph}$ & $\mathrm{N}$ & $\mathrm{Me}$ & Co & $\mathrm{NE}$ & $\mathrm{P}$ & $\mathrm{P}$ & \\
\hline
\end{tabular}

\section{ROSIDS}

Vitales Juss. ex Bercht. \& J.Presl.

Vitaeae Juss.

Ampelocissus tomentosa (Roth) Planch.

Causonis trifolia (L.) Mabb. \& J.Wen

Cayratia pedata (Lam.) Gagnep.

Cissus quinquangularis Chiov.

Fabales Bromhead

Fabaceae Lindl.

Abrus precatorius $\mathrm{L}$

Acacia auriculiformis A.Cunn. ex Benth.

Albizia lebbeck (L.) Benth.

Cassia fistula $\mathrm{L}$.

Chamaecrista mimosoides (L.) Greene

Crotalaria retusa $\mathrm{L}$.

Flemingia strobilifera (L.) W.T.Aiton Grona triflora (L.) H.Ohashi \& K.Ohashi

Guilandina bonduc L.

Mimosa pudica $\mathrm{L}$.

Mucuna pruriens (L.) DC.

Pithecellobium dulce (Roxb.) Benth.

Pseudarthria viscida (L.) Wight \& Arn.

Senna occidentalis (L.) Link

Senna siamea (Lam.) H.S.Irwin \& Barneby

Tephrosia pumila (Lam.) Pers.

Vigna vexillata (L.) A.Rich.

Zornia gibbosa Span.

Rosales Bercht. \& J.Presl

Rhamnaceae Juss.

Ventilago denticulata Willd.

Ziziphus nummularia (Burm.f.) Wight \& Arn.

Ziziphus oenopolia (L.) Mill.

Ulmaceae Mirb.

Holoptelea integrifolia (Roxb.) Planch

Moraceae Gaudich.

Ficus benghalensis L.

Streblus asper Lour.

Cucurbitales Juss. Ex Bercht. \& J. Presl

Cucurbitaceae Juss.

Cayaponia laciniosa (L.) C.Jeffrey

Coccinia grandis (L.) Voigt

Melothria trilobata Cogn.

Trichosanthes tricuspidata Lour.

Oxalidales Bercht. \& J. Presl

Oxalidaceae R. Br.

Oxalis corniculata L.

Malpighiales Juss. ex Bercht. \& J.Presl

Violaceae Batsch

Afrohybanthus enneaspermus (L.) Flicker

Salicaceae Mirb.

Flacourtia indica (Burm. f.) Merr.

Euphorbiaceae Juss.

Acalypha indica L.

$\begin{array}{llllcl}\text { USVT1 } & \text { C } & \text { P } & \text { N } & \text { Aug.-Dec. } & \text { Ph } \\ \text { USVT2 } & \text { C } & \text { P } & \text { N } & \text { Aug.-Dec. } & \text { Ph } \\ \text { USVT3 } & \text { C } & \text { P } & \text { N } & \text { Aug.-Feb. } & \text { Ph } \\ \text { USVT4 } & \text { C } & \text { P } & \text { N } & \text { Jul.-Jan. } & \text { Ph }\end{array}$

$\begin{array}{lllllll}\mathrm{N} & \mathrm{Me} & \mathrm{Sg} & \mathrm{NE} & \mathrm{P} & \mathrm{P} & \mathrm{P} \\ \mathrm{N} & \mathrm{No} & \mathrm{Co} & \mathrm{NE} & \mathrm{A} & \mathrm{P} & \mathrm{P} \\ \mathrm{N} & \mathrm{No} & \mathrm{Ov} & \mathrm{VU} & \mathrm{P} & \mathrm{P} & \mathrm{P} \\ \mathrm{N} & \mathrm{No} & \mathrm{Co} & \mathrm{NE} & \mathrm{P} & \mathrm{P} & \mathrm{P}\end{array}$

USFA1 $\quad \mathrm{C} \quad \mathrm{P} \quad \mathrm{N}$ Aug.-Mar. $\mathrm{Ph}$

$\begin{array}{llllll}\text { USFA2 } & \mathrm{T} & \mathrm{P} & \mathrm{N} & \text { Feb.-Oct. } & \mathrm{Ph}\end{array}$

$\begin{array}{llllll}\text { USFA3 } & \mathrm{T} & \mathrm{P} & \mathrm{N} & \text { Mar.-Feb. } & \mathrm{Ph}\end{array}$

$\begin{array}{lllll}\text { USFA4 } & \mathrm{T} & \mathrm{P} & \mathrm{N} & \text { Feb.-Dec. } \mathrm{Ph}\end{array}$

USFA5 H A N Mar.-Dec. Th

USFA6 $\mathrm{S}$ A E Jul.-Jan. Ch

$\begin{array}{lllll}\text { USFA7 } & \mathrm{H} & \mathrm{A} & \mathrm{N} & \text { Feb.-Sep. } \\ \mathrm{Ch}\end{array}$

$\begin{array}{llllll}\text { USFA8 } & \mathrm{H} & \mathrm{P} & \mathrm{N} & \text { Jul.-Feb. } & \mathrm{Th}\end{array}$

USFA9 $\mathrm{C} \quad \mathrm{P} \quad \mathrm{N}$ Aug.-Apr. Ph

USFA10 H P $\mathrm{H}$ Jul.-Nov. Th

$\begin{array}{lllll}\text { USFA11 } & \mathrm{C} & \mathrm{A} & \mathrm{N} & \text { Sep.-May } \\ \mathrm{Ph}\end{array}$

$\begin{array}{llllll}\text { USFA12 } & \mathrm{T} & \mathrm{P} & \mathrm{N} & \text { Feb.-Jul. } \mathrm{Ph}\end{array}$

$\begin{array}{lllll}\text { USFA13 } & \mathrm{H} & \mathrm{P} & \mathrm{N} & \text { Oct.- Jan. Th }\end{array}$

USFA14 S A E Aug.-Dec. Ch

$\begin{array}{llllll}\text { USFA15 } & T & P & E & \text { Sep.-Dec. } & P h\end{array}$

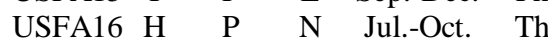

USFA17 C A $\mathrm{N}$ Jul.-Oct. Ph

USFA18 H A N Aug.-Nov. Th

$\begin{array}{llllll}\text { USRH1 } & \text { C } & \text { P } & \text { N } & \text { Nov.-Mar. } & \text { Ph } \\ \text { USRH2 } & \text { S } & \text { P } & \text { N } & \text { Sep.-Mar. } & \text { Ph } \\ \text { USRH3 } & \text { C } & \text { P } & \text { N } & \text { Nov.-Mar. } & \text { Ph }\end{array}$

$\begin{array}{lllllll}\mathrm{N} & \mathrm{Na} & \mathrm{Ob} & \mathrm{NE} & \mathrm{A} & \mathrm{P} & \mathrm{P}\end{array}$

$\begin{array}{lllllll}M & \mathrm{Me} & \mathrm{Ob} & \mathrm{LC} & \mathrm{P} & \mathrm{P} & \mathrm{P}\end{array}$

$\begin{array}{lllllll}\mathrm{MM} & \mathrm{Mi} & \mathrm{Ob} & \mathrm{NE} & \mathrm{P} & \mathrm{P} & \mathrm{P}\end{array}$

$\begin{array}{lllllll}\mathrm{N} & \mathrm{No} & \mathrm{Su} & \mathrm{LC} & \mathrm{P} & \mathrm{P} & \mathrm{P}\end{array}$

$\mathrm{Na}$ La LC A $\mathrm{P}$ A

$\begin{array}{llllll}\mathrm{Mi} & \mathrm{Ov} & \mathrm{NE} & \mathrm{P} & \mathrm{P} & \mathrm{P}\end{array}$

$\mathrm{Na}$ Ov NE A $\mathrm{P}$ P

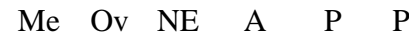

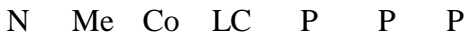

$\mathrm{Na}$ La LC A $\mathrm{P} \quad \mathrm{P}$

$\mathrm{N}$ No $\mathrm{Co}$ NE $\mathrm{A} \quad \mathrm{P} \quad \mathrm{P}$

M Mi Co LC $\mathrm{P} \quad \mathrm{P} \quad \mathrm{P}$

$\mathrm{Mi}$ Ov NE A P A

No Oo NE A $P$ P

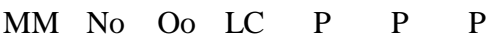

$\mathrm{Na}$ Oo LC A $\mathrm{P} \quad \mathrm{P}$

$\mathrm{N} \quad \mathrm{Mi} \quad \mathrm{Co}$ NE A $\mathrm{P}$ A

$\begin{array}{lllll}\mathrm{Na} & \mathrm{La} & \mathrm{NE} & \mathrm{A} & \mathrm{P}\end{array}$

$\begin{array}{lllllll}\mathrm{N} & \mathrm{Me} & \mathrm{La} & \mathrm{NE} & \mathrm{P} & \mathrm{P} & \mathrm{P}\end{array}$

$\begin{array}{lllllll}\mathrm{N} & \text { No } & \text { Ov } & \text { NE } & \text { P } & \text { P } & \text { P } \\ \mathrm{N} & \text { No } & \text { Ov } & \text { LC } & \text { P } & \text { P } & \text { P }\end{array}$

$\begin{array}{llllll}\text { USUL1 } & \mathrm{T} & \mathrm{P} & \mathrm{N} & \text { Jan.-Jun. } & \mathrm{Ph}\end{array}$

$\begin{array}{lllllll}\text { MM } & \mathrm{Me} & \mathrm{Ov} & \mathrm{NE} & \mathrm{P} & \mathrm{P} & \mathrm{P}\end{array}$

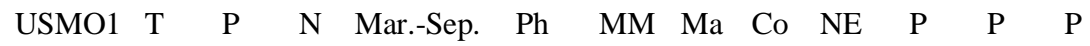

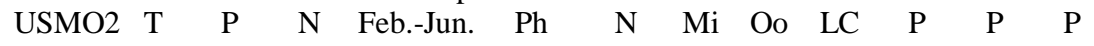

USCU1 C A $\mathrm{N}$ Jun.-Jan. $\mathrm{Ph}$

USCU2 C A $\mathrm{N}$ Mar.-Dec. Ph

USCU3 C A N Jul.-Feb. Ph

USCU4 C A $\quad$ N Apr.-Sep. Ph

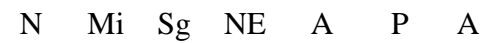

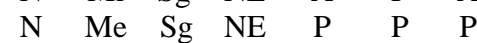

$\begin{array}{lllllll}\mathrm{N} & \mathrm{Me} & \mathrm{OV} & \mathrm{NE} & \mathrm{A} & \mathrm{P} & \mathrm{P}\end{array}$

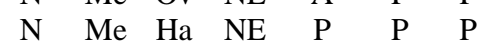

\begin{tabular}{cccccccccccc} 
USOX1 & H & A & E & All & Th & Na & Cu & NE & P & P & P \\
USVI1 & H & P & N & Jul.-Nov. & Th & Na & La & NE & A & P & A \\
USSA1 & S & P & N & Sep.-May. & Ch & Mi & Ov & LC & P & P & P \\
& & & & & & & & & & & \\
USEU1 & H & A & E & All & Th & No & Ov & NE & A & P & P \\
USEU2 & S & P & N & Jun.-Jan. & Ch & No & Ov & LC & p & p & p \\
\hline
\end{tabular}




\begin{tabular}{|c|c|c|c|c|c|c|c|c|c|c|c|c|c|}
\hline Chrozophora rottleri (Geiseler) Spreng. & USEU3 & $\mathrm{H}$ & A & $E$ & Jul.-Feb. & Th & & $\mathrm{Na}$ & $\mathrm{Co}$ & $\mathrm{NE}$ & A & $\mathrm{P}$ & $\mathrm{P}$ \\
\hline Croton bonplandianus Baill. & USEU4 & $\mathrm{H}$ & $\mathrm{P}$ & $\mathrm{E}$ & All & Th & & No & Co & $\mathrm{NE}$ & $\mathrm{P}$ & $\mathrm{P}$ & $\mathrm{P}$ \\
\hline Croton bonplandianus Baill. & USEU5 & $\mathrm{H}$ & $\mathrm{P}$ & $\mathrm{E}$ & All & Th & & No & $\mathrm{Ov}$ & $\mathrm{NE}$ & $\mathrm{P}$ & $\mathrm{P}$ & $\mathrm{P}$ \\
\hline Euphorbia antiquorum L. & USEU6 & $\mathrm{T}$ & $\mathrm{P}$ & $\mathrm{N}$ & Jan.-Apr. & $\mathrm{Ph}$ & $\mathrm{N}$ & Le & Oo & $\mathrm{NE}$ & $\mathrm{P}$ & $\mathrm{P}$ & $\mathrm{P}$ \\
\hline Euphorbia hirta L. & USEU7 & $\mathrm{H}$ & A & $\mathrm{E}$ & Feb.-Dec. & Th & & $\mathrm{Na}$ & $\mathrm{Co}$ & $\mathrm{NE}$ & A & $\mathrm{P}$ & $\mathrm{P}$ \\
\hline Jatropha gossypiifolia L. & USEU8 & $\mathrm{S}$ & $\mathrm{P}$ & $\mathrm{E}$ & Mar.-Aug. & $\mathrm{Ch}$ & & $\mathrm{Ma}$ & Oo & $\mathrm{LC}$ & $\mathrm{P}$ & $\mathrm{P}$ & $\mathrm{P}$ \\
\hline $\begin{array}{l}\text { Suregada multiflora (A.Juss.) Baill. } \\
\text { Phyllanthaceae Martinov }\end{array}$ & USEU9 & $\mathrm{T}$ & $\mathrm{P}$ & $\mathrm{N}$ & Mar.-Jul. & $\mathrm{Ph}$ & $\mathrm{N}$ & Mi & Ov & $\mathrm{NE}$ & $\mathrm{P}$ & $\mathrm{P}$ & $\mathrm{P}$ \\
\hline Breynia vitis-idaea (Burm.f.) C.E.C.Fisch. & USPY1 & $\mathrm{S}$ & $\mathrm{P}$ & $\mathrm{N}$ & Apr.-Dec. & $\mathrm{Ch}$ & & $\mathrm{Mi}$ & Ov & $\mathrm{LC}$ & $\mathrm{P}$ & $\mathrm{P}$ & $\mathrm{P}$ \\
\hline $\begin{array}{l}\text { Phyllanthus debilis J.G.Klein ex Willd. } \\
\text { Myrtales Juss. ex Bercht. \& J.Presl } \\
\text { Combretaceae R.Br. }\end{array}$ & USPY2 & $\mathrm{H}$ & A & $\mathrm{N}$ & Apr.-Sep. & Th & & Le & Ov & NE & A & $\mathrm{P}$ & $\mathrm{P}$ \\
\hline $\begin{array}{l}\text { Combretum indicum (L.) DeFilipps } \\
\text { Myrtaceae Juss. }\end{array}$ & USCO1 & $\mathrm{C}$ & $\mathrm{P}$ & $\mathrm{N}$ & All & $\mathrm{Ph}$ & $\mathrm{N}$ & $\mathrm{Me}$ & Ov & NE & $\mathrm{P}$ & $\mathrm{P}$ & $\mathrm{P}$ \\
\hline $\begin{array}{l}\text { Syzygium cumini (L.) Skeels } \\
\text { Sapindales Juss. Ex Bercht. \& J.Presl } \\
\text { Anacardiaceae R.Br. }\end{array}$ & USMY1 & $\mathrm{T}$ & $\mathrm{P}$ & $\mathrm{N}$ & Mar.-Jul. & $\mathrm{Ph}$ & MM & $\mathrm{Me}$ & $\mathrm{La}$ & $\mathrm{LC}$ & $\mathrm{P}$ & $\mathrm{P}$ & $\mathrm{P}$ \\
\hline $\begin{array}{l}\text { Mangifera indica } \mathrm{L} \text {. } \\
\text { Sapindaceae Juss. }\end{array}$ & USAN1 & $\mathrm{T}$ & $\mathrm{P}$ & $\mathrm{N}$ & Feb.- Jun. & $\mathrm{Ph}$ & M & $\mathrm{Ma}$ & $\mathrm{Ob}$ & DD & $\mathrm{P}$ & $\mathrm{P}$ & $\mathrm{P}$ \\
\hline Allophylus cobbe (L.) Forsyth f. & USSP1 & $\mathrm{C}$ & $\mathrm{P}$ & $\mathrm{N}$ & Jul.-Oct. & $\mathrm{Ph}$ & M & No & Ov & $\mathrm{NE}$ & A & $\mathrm{P}$ & A \\
\hline Cardiospermum halicacabum $\mathrm{L}$. & USSP2 & $\mathrm{C}$ & A & $\mathrm{N}$ & Jul.-Dec. & $\mathrm{Ph}$ & $\mathrm{N}$ & No & $\mathrm{Sp}$ & $\mathrm{LC}$ & A & $\mathrm{P}$ & $\mathrm{P}$ \\
\hline $\begin{array}{l}\text { Dodonaea viscosa Jacq. } \\
\text { Rutaceae Juss. }\end{array}$ & USSP3 & $\mathrm{S}$ & $\mathrm{P}$ & $\mathrm{N}$ & Nov.-Apr. & $\mathrm{Ph}$ & $\mathrm{N}$ & No & $\mathrm{Ob}$ & $\mathrm{LC}$ & A & $\mathrm{P}$ & $\mathrm{P}$ \\
\hline Aegle marmelos (L.) Corrêa & USRU1 & $\mathrm{T}$ & $\mathrm{P}$ & $\mathrm{N}$ & May-Jul. & $\mathrm{Ph}$ & M & $\mathrm{Me}$ & $\mathrm{Ov}$ & NT & $\mathrm{P}$ & $\mathrm{P}$ & $\mathrm{P}$ \\
\hline $\begin{array}{l}\text { Murraya paniculata (L.) Jack } \\
\text { Simaroubaceae DC. }\end{array}$ & USRU2 & $\mathrm{S}$ & $\mathrm{P}$ & $\mathrm{N}$ & Apr.-Jun. & $\mathrm{Ph}$ & $\mathrm{N}$ & $\mathrm{Na}$ & Ov & $\mathrm{NE}$ & $\mathrm{P}$ & $\mathrm{P}$ & $\mathrm{P}$ \\
\hline $\begin{array}{l}\text { Ailanthus excelsa Roxb. } \\
\text { Meliaceae Juss. }\end{array}$ & USSI1 & $\mathrm{T}$ & $\mathrm{P}$ & $\mathrm{N}$ & Jan.-Oct. & $\mathrm{Ph}$ & MM & $\mathrm{Ma}$ & $\mathrm{Ob}$ & $\mathrm{NE}$ & $\mathrm{P}$ & $\mathrm{P}$ & $\mathrm{P}$ \\
\hline Azadirachta indica A. Juss. & USML1 & $\mathrm{T}$ & $\mathrm{P}$ & $\mathrm{N}$ & Mar.-Jul. & $\mathrm{Ph}$ & M & No & $\mathrm{La}$ & $\mathrm{LC}$ & $\mathrm{P}$ & $\mathrm{P}$ & $\mathrm{P}$ \\
\hline $\begin{array}{l}\text { Melia azedarach L. } \\
\text { Malvales Juss. ex Bercht. \& J.Presl } \\
\text { Malvaceae Juss. }\end{array}$ & USML2 & $\mathrm{T}$ & $\mathrm{P}$ & $\mathrm{E}$ & Feb.-Nov. & $\mathrm{Ph}$ & M & No & $\mathrm{La}$ & $\mathrm{LC}$ & $\mathrm{P}$ & $\mathrm{P}$ & $\mathrm{P}$ \\
\hline Abutilon indicum (L.) Sweet & USMA1 & $\mathrm{S}$ & A & $\mathrm{N}$ & Sep.-Apr. & $\mathrm{Ch}$ & & $\mathrm{Ma}$ & Co & NE & A & $\mathrm{P}$ & $\mathrm{P}$ \\
\hline Azanza lampas (Cav.) Alef. & USMA2 & $\mathrm{S}$ & A & $\mathrm{N}$ & Sep.-Dec. & $\mathrm{Ch}$ & & No & $\mathrm{Sg}$ & NE & $\mathrm{P}$ & $\mathrm{P}$ & $\mathrm{P}$ \\
\hline Byttneria herbacea Roxb. & USMA3 & $\mathrm{H}$ & A & $\mathrm{N}$ & Sep.-Nov. & Th & & No & $\mathrm{Co}$ & $\mathrm{NE}$ & A & $\mathrm{P}$ & $\mathrm{P}$ \\
\hline Corchorus aestuans L. & USMA4 & $\mathrm{H}$ & A & $\mathrm{E}$ & Jul.-Nov. & $\mathrm{Th}$ & & $\mathrm{Me}$ & Ov & $\mathrm{NE}$ & A & $\mathrm{P}$ & A \\
\hline Malachra capitata (L.) L. & USMA5 & $\mathrm{H}$ & A & $\mathrm{E}$ & Sep.-Nov. & Th & & Mi & $\mathrm{Ha}$ & $\mathrm{NE}$ & A & $\mathrm{P}$ & A \\
\hline Melochia corchorifolia $\mathrm{L}$. & USMA6 & $\mathrm{H}$ & A & $\mathrm{E}$ & May.-Jun. & $\mathrm{Th}$ & & $\mathrm{Na}$ & Co & $\mathrm{LC}$ & A & $\mathrm{P}$ & A \\
\hline Sida acuta Burm.f. & USMA7 & $\mathrm{S}$ & A & $\mathrm{N}$ & Aug.-Dec. & $\mathrm{Th}$ & & No & Co & $\mathrm{NE}$ & A & $\mathrm{P}$ & $\mathrm{P}$ \\
\hline Sida cordifolia $\mathrm{L}$. & USMA8 & $\mathrm{S}$ & A & $\mathrm{N}$ & Aug.-Dec. & Th & & $\mathrm{Mi}$ & $\mathrm{Co}$ & $\mathrm{NE}$ & A & $\mathrm{P}$ & A \\
\hline $\begin{array}{l}\text { Urena lobata } \mathrm{L} \text {. } \\
\text { Brassicales Bromhead } \\
\text { Capparaceae Juss. }\end{array}$ & USMA9 & $\mathrm{S}$ & A & $\mathrm{E}$ & Sep.-Dec. & $\mathrm{Ch}$ & & No & $\mathrm{Ha}$ & $\mathrm{LC}$ & A & $\mathrm{P}$ & $\mathrm{P}$ \\
\hline $\begin{array}{l}\text { Capparis zeylanica L. } \\
\text { Cleomaceae Bercht. \& J.Presl }\end{array}$ & USCP1 & $\mathrm{C}$ & $\mathrm{P}$ & $\mathrm{N}$ & Mar.-Oct. & $\mathrm{Ph}$ & M & No & $\mathrm{La}$ & $\mathrm{NE}$ & $\mathrm{P}$ & $\mathrm{P}$ & $\mathrm{P}$ \\
\hline Cleome monophylla $\mathrm{L}$. & USCE1 & $\mathrm{H}$ & A & $\mathrm{E}$ & Aug.-Oct. & Th & & $\mathrm{Mi}$ & $\mathrm{Co}$ & $\mathrm{NE}$ & A & $\mathrm{P}$ & $\mathrm{P}$ \\
\hline $\begin{array}{l}\text { Cleome viscosa } \mathrm{L} \text {. } \\
\text { SUPERASTERIDS }\end{array}$ & USCE2 & $\mathrm{H}$ & A & $\mathrm{E}$ & Sep.-Apr. & Th & & No & $\mathrm{Ob}$ & NE & A & $\mathrm{P}$ & $\mathrm{P}$ \\
\hline $\begin{array}{l}\text { Santalales R.Br. ex Bercht. \& J.Presl } \\
\text { Loranthaceae Juss. }\end{array}$ & & & & & & & & & & & & & \\
\hline $\begin{array}{l}\text { Macrosolen capitellatus (Wight \& Arn.) Danser } \\
\text { Caryophyllales Juss. ex Bercht. \& J.Presl } \\
\text { Polygonaceae Juss. }\end{array}$ & USLO1 & $\mathrm{S}$ & A & $\mathrm{N}$ & Mar.-Sep. & $\mathrm{Ph}$ & $\mathrm{N}$ & No & $\mathrm{Li}$ & $\mathrm{NE}$ & A & $\mathrm{P}$ & $\mathrm{P}$ \\
\hline $\begin{array}{l}\text { Persicaria hydropiper (L.) Delarbre } \\
\text { Caryophyllaceae Juss. }\end{array}$ & USPL1 & $\mathrm{H}$ & A & $\mathrm{N}$ & May-Jan. & Th & & $\mathrm{Na}$ & $\mathrm{La}$ & $\mathrm{LC}$ & A & $\mathrm{P}$ & $\mathrm{P}$ \\
\hline $\begin{array}{l}\text { Vaccaria hispanica (Mill.) Rauschert } \\
\text { Amaranthaceae Juss }\end{array}$ & USCR1 & $\mathrm{H}$ & A & $\mathrm{N}$ & Jan.-Mar. & Th & & $\mathrm{Le}$ & $\mathrm{Su}$ & $\mathrm{NE}$ & A & $\mathrm{P}$ & A \\
\hline Achyranthes aspera L. & USAM1 & $\mathrm{H}$ & A & $\mathrm{N}$ & Sep.-Feb. & Th & & Mi & Ov & $\mathrm{NE}$ & A & $\mathrm{P}$ & A \\
\hline Alternanthera sessilis (L.) R. Br. ex DC. & USAM2 & $\mathrm{H}$ & A & $\mathrm{E}$ & Jul.-Feb. & Th & & Mi & Ov & $\mathrm{LC}$ & $\mathrm{P}$ & $\mathrm{P}$ & $\mathrm{P}$ \\
\hline Amaranthus spinosus $\mathrm{L}$. & USAM3 & $\mathrm{H}$ & A & $\mathrm{E}$ & All & Th & & $\mathrm{Na}$ & Ov & $\mathrm{NE}$ & $\mathrm{P}$ & $\mathrm{P}$ & $\mathrm{P}$ \\
\hline $\begin{array}{l}\text { Ouret lanata (L.) Kuntze } \\
\text { Nyctaginaceae Juss. }\end{array}$ & USAM4 & $\mathrm{H}$ & A & $\mathrm{E}$ & Nov.-Jan. & $\mathrm{Th}$ & & Le & Ov & $\mathrm{NE}$ & A & $\mathrm{P}$ & $\mathrm{P}$ \\
\hline $\begin{array}{l}\text { Boerhavia diffusa L. } \\
\text { Portulacaceae Juss. }\end{array}$ & USNC1 & $\mathrm{H}$ & A & $\mathrm{N}$ & Jun.-Dec. & $\mathrm{Th}$ & & Mi & $\mathrm{Re}$ & $\mathrm{NE}$ & A & $\mathrm{P}$ & A \\
\hline $\begin{array}{l}\text { Portulaca oleracea L. } \\
\text { Cactaceae Juss. }\end{array}$ & USPR1 & $\mathrm{H}$ & A & $\mathrm{E}$ & Jun.-Dec. & $\mathrm{Th}$ & & Mi & Oo & $\mathrm{NE}$ & $\mathrm{P}$ & $\mathrm{P}$ & $\mathrm{P}$ \\
\hline Opuntia stricta (Haw.) Haw. & USCC1 & $\mathrm{S}$ & $\mathrm{P}$ & $\mathrm{E}$ & Apr.-Aug. & $\mathrm{Ch}$ & & Le & Ac & $\mathrm{LC}$ & $\mathrm{P}$ & $\mathrm{P}$ & $\mathrm{P}$ \\
\hline
\end{tabular}




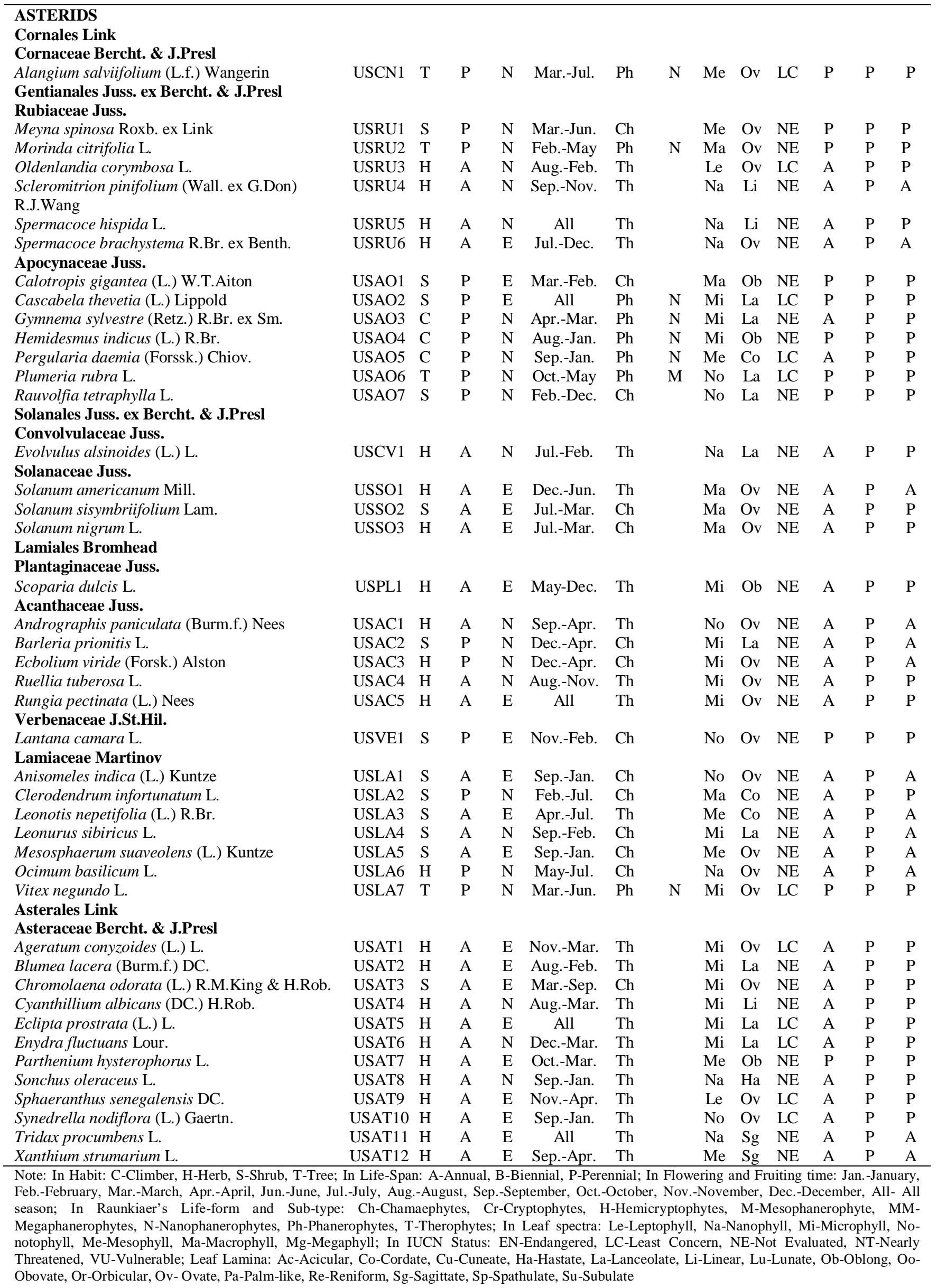


Table 2. Total angiosperm taxa

\begin{tabular}{lcccccccc}
\hline \multirow{2}{*}{ Group } & \multirow{2}{*}{ Orders } & \multirow{2}{*}{ Families } & \multirow{2}{*}{ Genera } & \multicolumn{3}{c}{ Species } \\
\cline { 5 - 9 } & & & & Herbs & Shrubs & Trees & Climber & Total \\
\hline Dicots & 19 & 41 & 121 & 54 & 29 & 21 & 26 & 130 \\
Monocots & 7 & 10 & 27 & 25 & 1 & 2 & 4 & 32 \\
Total & 26 & 51 & 148 & 79 & 30 & 23 & 30 & 162 \\
\hline
\end{tabular}

25

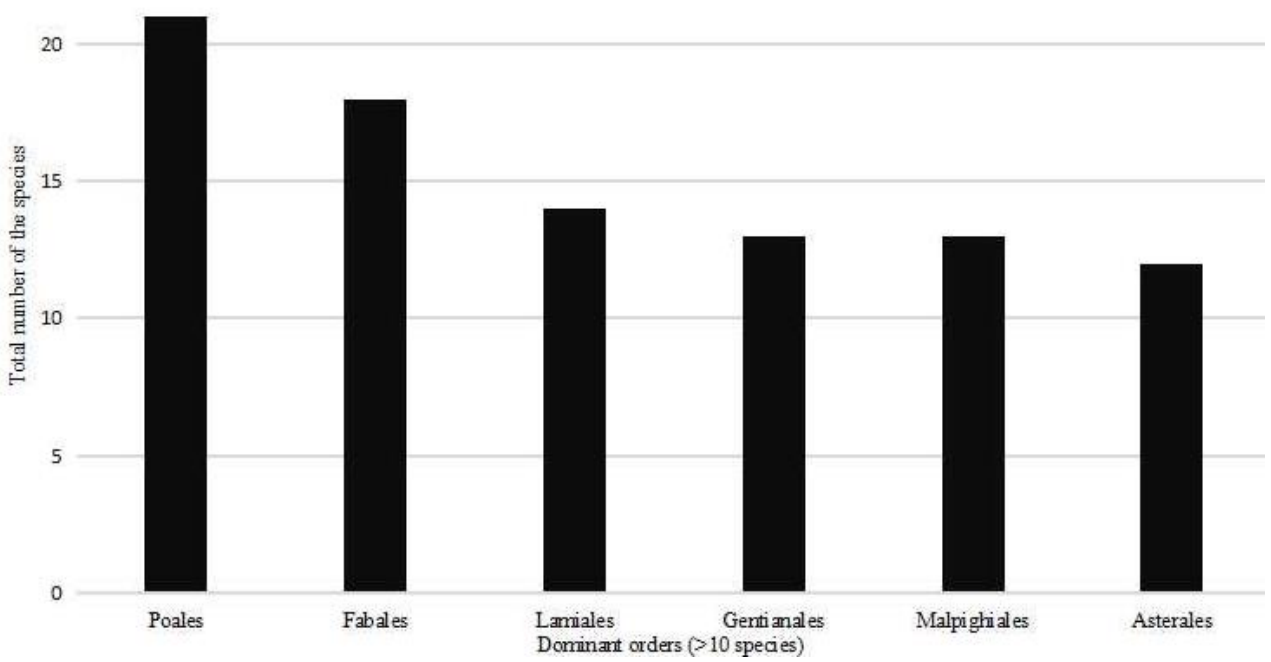

Figure 2. Dominant orders ( $>10$ species) of the sacred grove

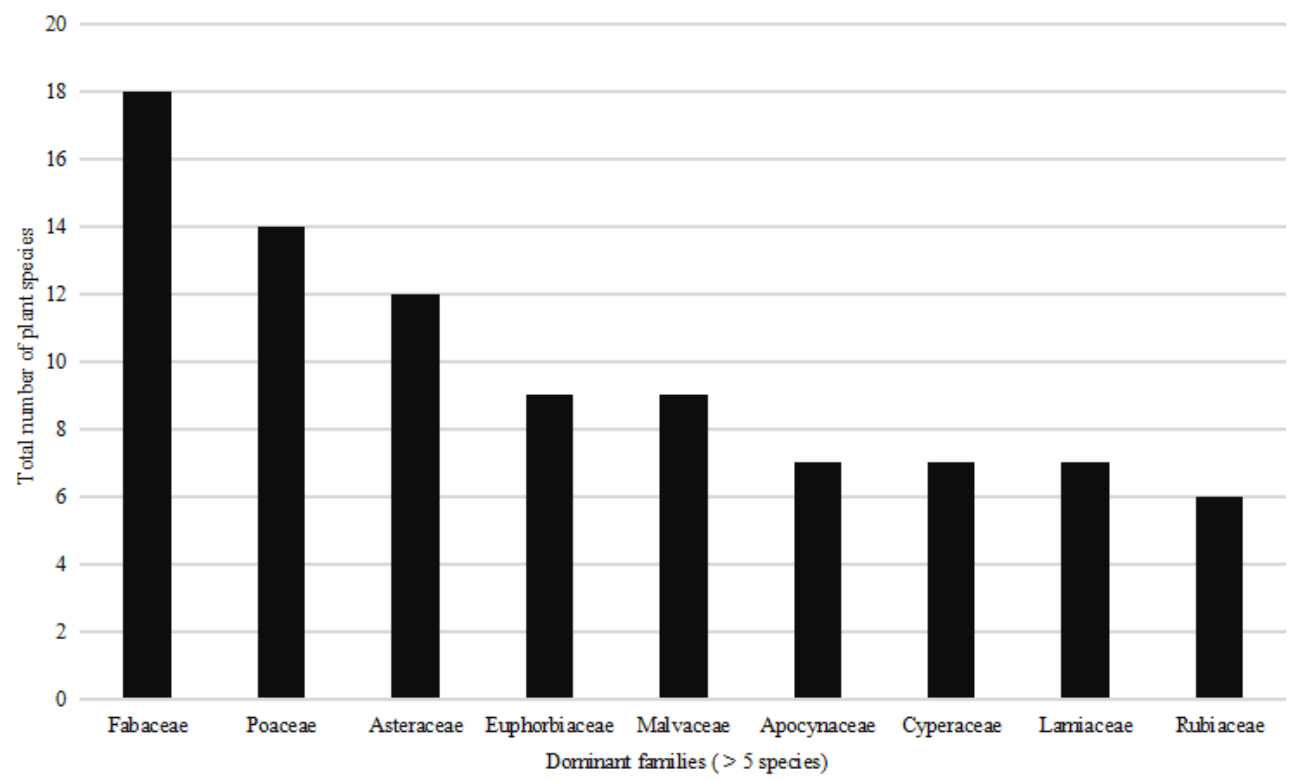

Figure 3. Dominant families ( $>5$ species) of the sacred grove 


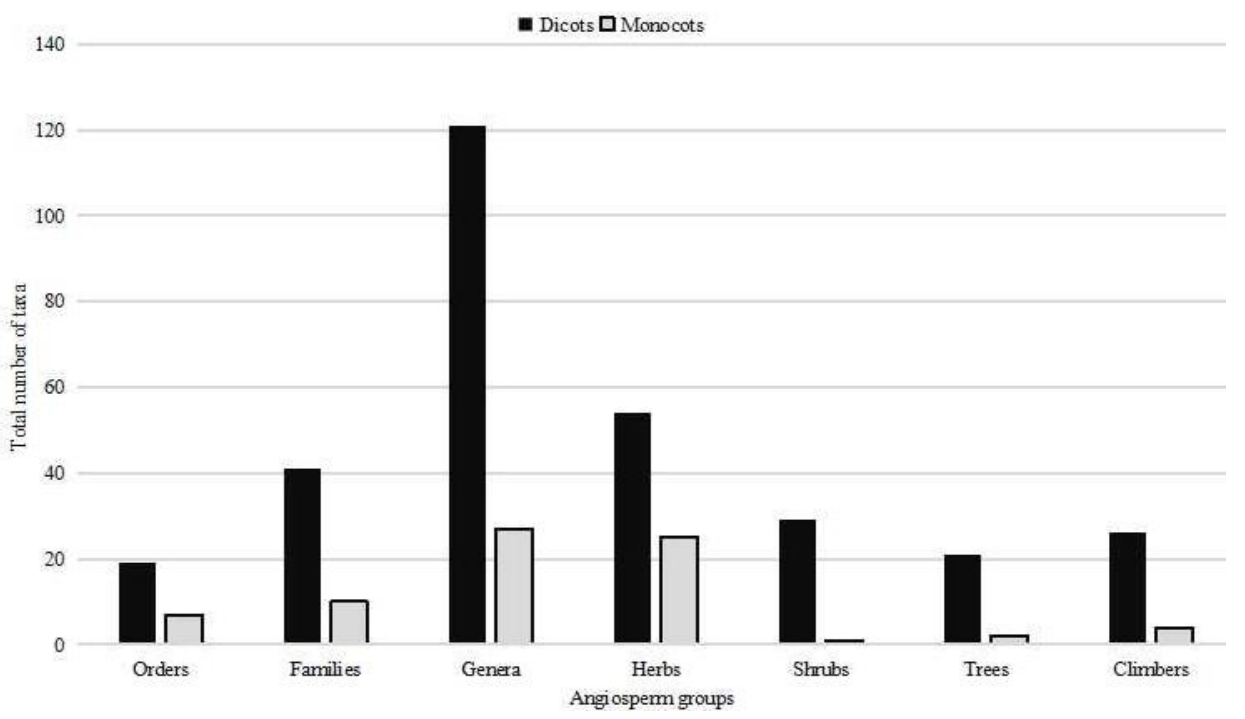

Figure 4. Total angiosperm taxa

Therefore, common invasive species growing in the grove were herbs (Alternanthera sessilis) and shrubs (Chromolaena odorata, Mesosphaerum suaveolens, Lantana camara). In recent years, unsustainable anthropogenic activities in this grove have been regarded as a severe threat to the local vegetation (Blondel 2006). Exotic species had already started to naturalize in the study region, especially in newly created forest gaps and degraded areas. It was observed during the present study that alien plants $(C$. odorata, $M$. suaveolens) spread very rapidly in the gaps in the forest (Martin et al. 2009). Other alien species discovered during the current investigation in the region are expected to become essential plant invaders shortly, necessitating immediate attention and management.

\section{Life form and biological spectrum}

The biological spectrum showed that 55 Phanerophytes (33.95\%) were the most frequent, followed by 53 Therophytes (32.72\%), 26 Chamaephytes (16.05\%), 21 Hemicryptophytes (12.96\%) and 7 Cryptophytes (4.32\%). Of the Phanerophytes, 38 Nanophanerophytes $(23.46 \%)$ were more common than 11 Mesophanerophytes (6.79\%) and 6 Megaphanerophytes (3.70\%) (Table 3).

Therophytes and Chamaephytes had shown a greater percentage of $19.72 \%$ and $7.05 \%$, respectively, than the normal spectrum of the "Thero-chamaephyte" phytoclimate. Further, the number of Hemicryptophytes (13.04\%), Phanerophytes (12.05\%), and Cryptophytes $(1.68 \%)$ were comparatively smaller in percentage than the normal spectrum. It was also noted that the Phanerophytes, Nanophanerophytes $(8.46 \%)$ were somewhat larger, and Mesophanerophytes (21.21\%) and Megaphanerophytes $(0.70 \%)$ were comparatively smaller in values than the normal spectrum (Table 3, Figure 5).

The observed flora was compared to Raunkiaer's (1934) standard range, created for world flora, and reflected homogeneous climatic conditions (Sharma and Raina
2017). The $\mathrm{c} 2$ test demonstrated a significant difference (c2 $=45.603, \mathrm{df}=4, \mathrm{P}<0.0001)$ between the observed flora and Raunkiaer's normal spectrum, which meant this difference was statistically highly significant (de Paula et al. 2017) (Table 3). The Phanerophytes' life form had the highest, partly due to the local protection under certain taboos of the sacred grove.

The highest percentage of therophytes in the area may be related to the subtropic character, which is often associated with various factors such as the region's soil and climatic conditions, combined with anthropogenic activities such as grazing, lopping, felling, deforestation, the introduction of annual weeds, etc. (Sharma and Raina 2017). Thus, the present study revealed that the vegetation was predominantly of sub-tropical type, with a higher percentage of Therophytes and Chamaephytes than the normal biological spectrum. Therophyte prevalence was also an indicator of biotic pressure. Therophyte development is reported to prefer in disturbed areas (AlYemeni and Sher 2010; Zemmar et al. 2020; Nafeesa et al. 2021; Haq et al. 2021). Our findings agree with them as the Therophytes dominated in the present area under investigation, which had hot, dry, and waterlogged conditions in parts combined with overgrazing, resulting in harsh conditions. Nazir and Malik (2006) reported that Nanophanerophytes and Therophytes were the biological spectra of Sarsawa Hill Kotli. The prevalence of Therophytes was also expressed in the present study.

The results also agree with Sher and Khan (2007) and Kar et al. (2010), who also argued that Therophytes and Nanophanerophytes are the characteristics of sub-tropical ecosystems. Structurally and floristically, the sub-tropical dry forests are less complex than wet forests, comprising about half or less of the moist forest tree species (Hasnat and Hossain 2020). The forests constituted a refuge for valuable and endangered plants and animals. Further study is necessary to quantify the data and suggest plans to conserve the sacred groves. 
Table 3. Biological spectrum ( $\%$ of all life forms) of the sacred grove and its comparison with Raunkiaer's normal spectrum

\begin{tabular}{lcccc}
\hline \multicolumn{1}{c}{ Life forms } & $\begin{array}{c}\text { Total no. } \\
\text { of species }\end{array}$ & $\begin{array}{c}\text { Biological spectrum (\%) } \\
\text { of the sacred grove }\end{array}$ & $\begin{array}{c}\text { Raunkiaer's normal } \\
\text { spectrum }(\%)\end{array}$ & $\begin{array}{c}\text { Deviation= (Raunkiaer's normal } \\
\text { spectrum- Biological spectrum) }\end{array}$ \\
\hline Phanerophytes (Ph) & 55 & 33.95 & 46.00 & -12.05 \\
Megaphanerophytes (MM) & 6 & 3.70 & 3.00 & +0.70 \\
Mesophanerophyte (M) & 11 & 6.79 & 28.00 & -21.21 \\
Nanophanerophytes (N) & 38 & 23.46 & 15.00 & +8.46 \\
Chamaephytes (Ch) & 26 & 16.05 & 9.00 & +7.05 \\
Hemicryptophytes (He) & 21 & 12.96 & 26.00 & -13.04 \\
Cryptophytes (Cr) & 7 & 4.32 & 6.00 & -1.68 \\
Therophytes (Th) & 53 & 32.72 & 13.00 & +19.72 \\
Total & 162 & 100.00 & 100 & \\
\hline
\end{tabular}

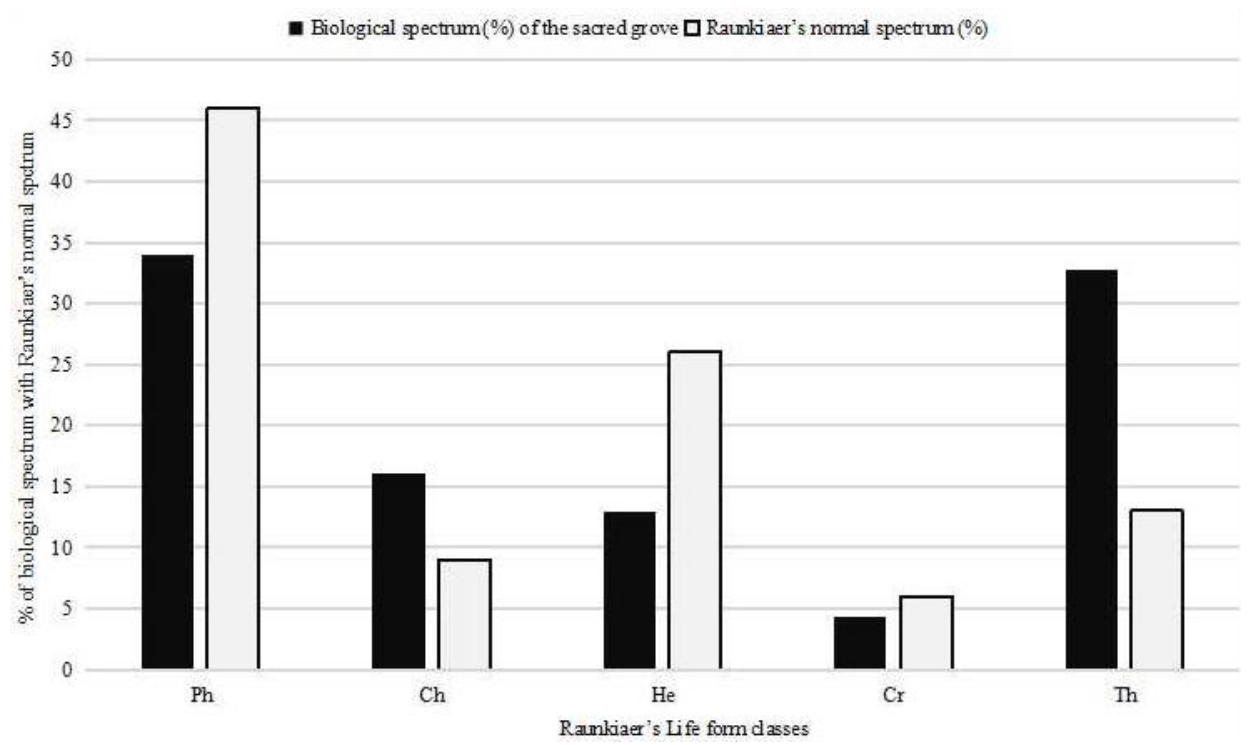

Figure 5. Comparison of the biological spectrum with Raunkiaer's normal spectra

\section{Leaf size spectra, leaf lamina, and phenology}

The overall leaf size spectrum shows that there were Leptophyllous 26 (16.05\%), Nanophyllous 24 (14.81\%), Microphyllous 35 (21.60\%), Notophyllous 33 (20.37\%), Mesophyllous 29 (17.90\%), Macrophyllous 14 (8.64\%) and Megaphyllous $1(0.62 \%)$ plants. Concerning the range of leaf sizes, microphyll was largely followed by Notophyll, Mesophyll, Leptophyll, Nanophyll, Macrophyll, and Megaphyll. Poaceae $12(7.41 \%)$ was the dominant Leptophyllous family, whereas Fabaceae 6 (3.70\%), Asteraceae 6 (3.70\%), Menispermaceae 5 (3.09\%), and Solanaceae $3(1.85 \%)$ were the major Mesophyllous, Microphyllous, Mesophyllous, and Macrophyllous families respectively. Whereas Notophyllous $4(2.47 \%)$ was equally distributed in Euphorbiaceae, Fabaceae, and Malvaceae families, and Asparagaceae $1(0.62 \%)$ was the only Megaphyllous family (Table 1 and 4, Figure 6).

The leaf spectrum revealed information about a community's plant adaption and association. Small-sized leaves were present at the base, while the large ones were at higher altitudes and correlated with climatic warming and water availability in the soil (Badshah et al. 2016). The median type of leaf size indicated the presence of subtropical climate type. Mesophyll, Notophyll, and Microphyllous elements were dominant in the sacred grove, which meant moisture availability or wet condition. In the present study, due to Therophytes and Chamaephytes, the proportion of different leaf size groups was observed to change seasonally (Haq et al. 2019). However, the Phanerophytes and some Chamaephytes preserved almost the same status in all seasons.

As regards the shape of leaf lamina, ovate 49 (30.25\%) was found to be the maximum, followed by cordate 24 (14.81\%), lanceolate $23(14.20 \%)$, acicular $13(8.02 \%)$, linear $12(7.41 \%)$, oblong $11(6.79 \%)$, obovate $7(4.32 \%)$, sagittate $7(4.32 \%)$, subulate $5(3.08 \%)$, hastate $4(2.47 \%)$, spathulate $3(1.85 \%)$, cuneate $1(0.62 \%)$, orbicular 1 $(0.62 \%)$, palmate $1(0.62 \%)$ and reniform $1(0.62 \%)$ (Table 1, Figure 6). Leaf spectra tell us about the community's plant adaptation and association. Small leaves were present at the base, whereas the broad leaves were present upwards, which correlated with climate warming and water availability in the soil (Wright et al. 2017). The medium leaf size revealed the sub-tropical climate. In the sacred grove, meso- and microphyllous elements were dominant, showing moisture and perennial water availability or wet 
condition (Sen and Bhakat 2021a). Leaf laminae in the sacred grove came in various shapes and sizes, ranging from oval to lunate. The leaf shape helps plants optimize light capture and water loss and minimizes the avoidable energy consumption in leaf production (Cowling and Field 2003). The distribution of leaf sizes in different resourcepoor environments strongly supports this theory (Reich et al. 2003). Not only did the leaf laminas differ in size, but certainly, leaves also had distinct serrations, and the blades of some leaves were almost fully dissected. Leaf dissection aids plants in reducing self-shading and, as a result, ensures that nearby leaves receive adequate light (Westoby et al. 2002).

The vegetation phenology observed during different seasons revealed that most of the species were dominant in rainy seasons $161(99.38 \%)$, followed by winter 124 $(76.54 \%)$ and summer $66(40.74 \%)$. Habit-wise species content varied seasonally; in the summer season, Tree $>$ Climber $>$ Shrub $>$ Herb; rainy season, Herb $>$ Shrub/Climber $>$ Tree; winter season, Herb> Climber $>$ Shrub $>$ Tree, respectively (Table 1, Figure 7). Variations in species composition within the community accompanied seasonal changes. The vegetation phenology recorded over different seasons demonstrated a substantial variance in vegetation, owing to the study region's welldefined seasons. Expectedly, it may be attributed to a high proportion of therophytes, cryptophytes, and chamaephytes in the region that appeared during the rainy and winter seasons (Haq et al. 2019).

\section{IUCN categories}

Among these 162 plants, 111 plants have not been evaluated till now. There were 48 Least Concerned (LC), 1 Nearly Threatened (NT), 1 Data Deficient (DD), and 1
Vulnerable (VU) species. Aegle marmelos was the Nearly Threatened tree species, whereas climber Cayratia pedata was the vulnerable species according to the IUCN (2021) (Table 1). The result of the threatened species assessment showed that most of them belong to perennial tree species. When the present phytosociological study was compared with the IUCN Red Listed data, it was observed that those plants were still present in the sacred groves with total regenerative capacity. Still, as per the local people's concept, those plants gradually disappeared in nearby areas. This study highlights the status and distribution of the species in the study area, the ecological characteristics required for their survival, and the threats to some species identified by the IUCN (2021) criteria. Various factors may be ascribed to habitat disturbance and vulnerability within the space, including anthropogenic activities, overgrazing which had a great negative impact on seedlings, and peripheral loss of land.

Table 4. Life-form analysis with different leaf size

\begin{tabular}{lcccccccc}
\hline Raunkiaer's & \multicolumn{7}{c}{ Leaf spectra } & Total \\
\cline { 2 - 9 } life form & Le & Na & Mi & No & Me & Ma & Mg & \\
\hline $\mathrm{Ph}$ & 1 & 2 & 11 & 17 & 20 & 4 & & 55 \\
$M M$ & & & 1 & 1 & 2 & 2 & 6 \\
$M$ & & & 2 & 5 & 3 & 1 & 11 \\
$N$ & 1 & 2 & 8 & 11 & 15 & 1 & & 38 \\
$\mathrm{Ch}$ & 1 & 2 & 7 & 7 & 2 & 6 & 1 & 26 \\
$\mathrm{He}$ & 18 & 2 & & 1 & & & & 21 \\
$\mathrm{Cr}$ & 1 & 1 & 1 & & 2 & 2 & & 7 \\
$\mathrm{Th}$ & 5 & 17 & 16 & 8 & 5 & 2 & & 53 \\
Total & 26 & 24 & 35 & 33 & 29 & 14 & 1 & 162 \\
\hline & & & & & & &
\end{tabular}

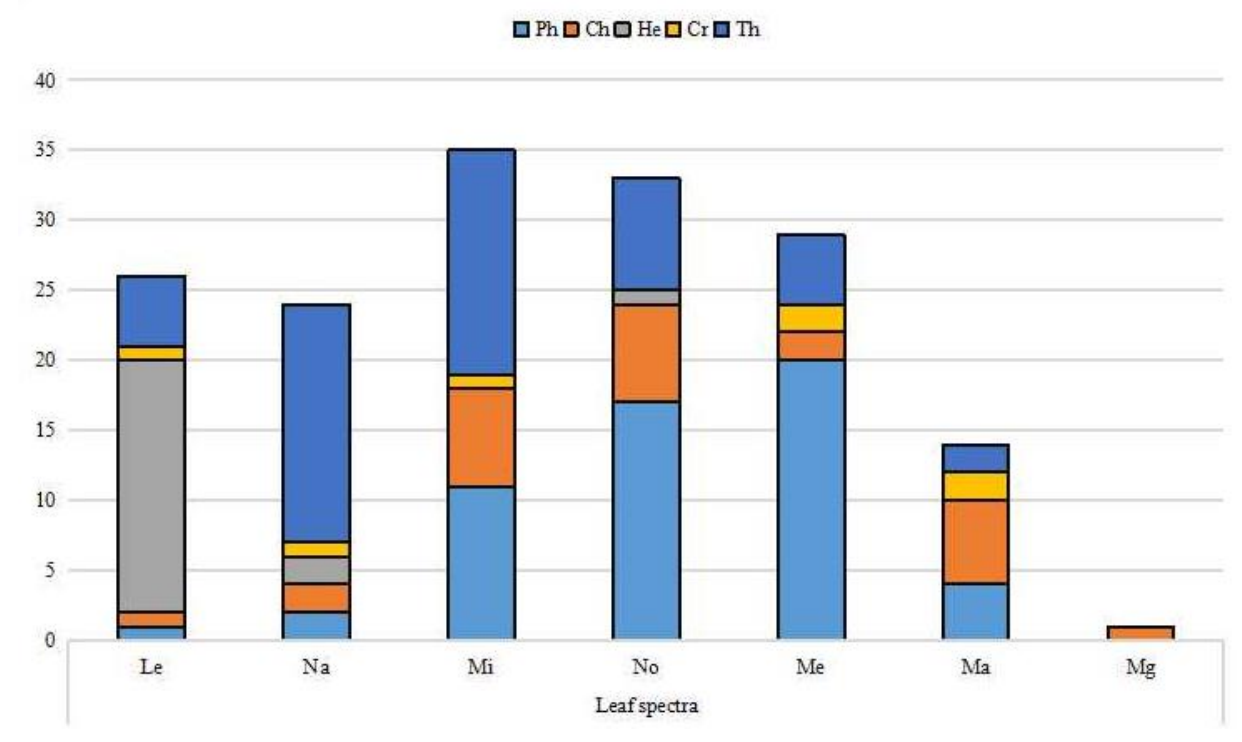

Figure 6. Analysis of life forms with different leaf sizes 


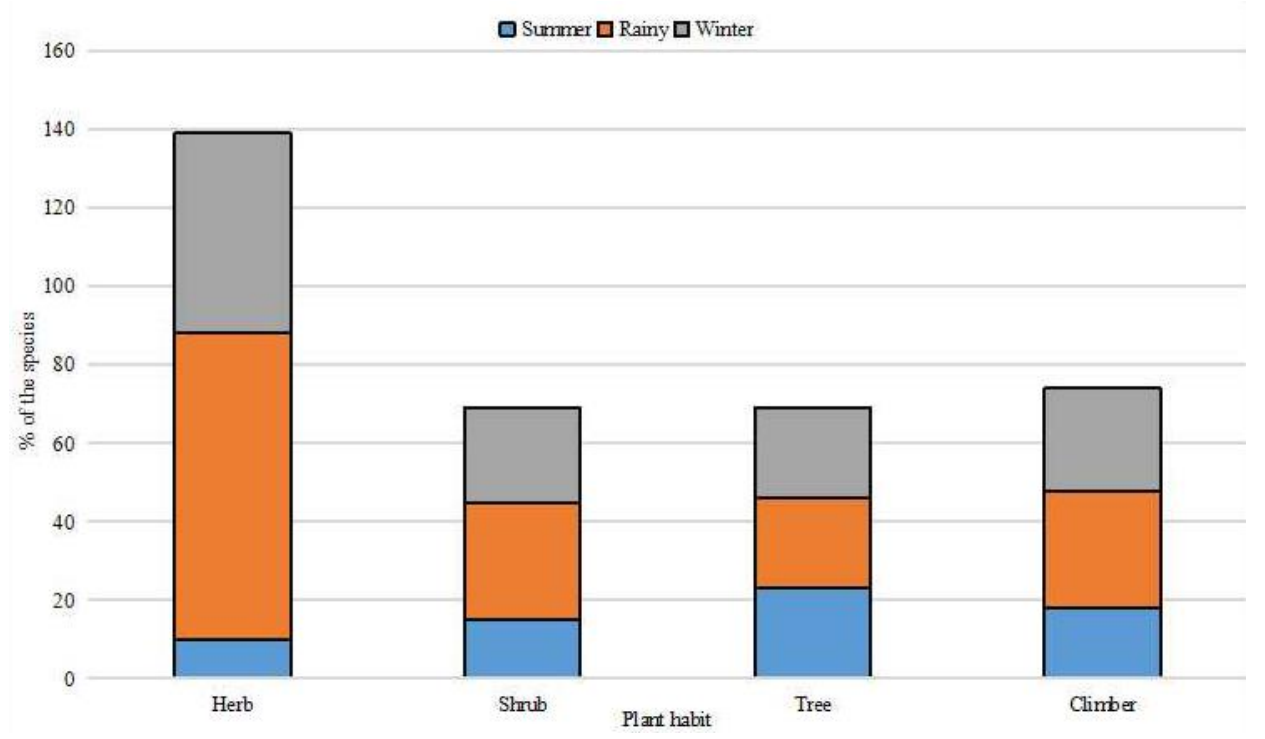

Figure 7. Vegetation phenology of the study area

In conclusion, the importance of cultural linkages in perpetuating the sacred groves has been well established. Indeed, the groves continued existence throughout millennia is a testament to the belief system's persistence. Finally, the current study has confirmed the presence of well-preserved sacred groves among the urban societies of West Bengal in Paschim Medinipur district. The study also demonstrates the importance of belief systems in preserving the integrity of the sacred grove in the face of modernism. According to the conservation values, these groves could serve as the nucleus for micro-level biodiversity protection. However, immediate and robust management measures are required to protect these biodiversity islands.

\section{ACKNOWLEDGEMENTS}

Special thanks to all informants who generously shared their knowledge of local plants and traditional medicinal uses. We are grateful for the proper identification of plant species by Dr. G. G. Maity, ex-Professor at Kalyani University. The authors declare that they have no known competing financial interests or personal relationships that could have influenced the work reported in this paper. Finally, we would like to thank the anonymous reviewers for carefully reading our manuscript and for their insightful comments and suggestions.

\section{REFERENCES}

Al-Yemeni M, Sher H. 2010. Biological spectrum with some other ecological attributes of the flora and vegetation of the Asir Mountain of Southwest, Saudi Arabia. Afr J Biotechnol 9 (34): 5550-5559.

Aniah P, Yelfaanibe A. 2016. Learning from the past: The role of sacred groves and shrines in environmental management in the Bongo district of Ghana. Environ Earth Sci 75 (10): 916-925. DOI: $10.1007 / \mathrm{s} 12665-016-5706-2$.
Anon. 2010. Medicinal Plant Resources of Southwest Bengal. Vol.-2. Research Wing, Directorate of Forests. Government of West Bengal, Kolkata, India.

Anon. 2011. District Human Development Report, Paschim Medinipur. Development \& Planning Department, Government of West Bengal, India. Retrieved from http://www.wbpspm.gov.in/SiteFiles/Publications/13_210620171032 39.pdf, accessed on 18 September, 2021.

Asif M, Iqbal Z, Alam J, Majid A, Ijaz F, Ali N et al. 2020. Floristic inventory and biological spectra of Balakot, District Mansehra, Pakistan. Acta Ecologica Sinica 40 (3): 197-203. DOI: 10.1016/j.chnaes.2019.05.009.

Badshah L, Hussain F, Sher Z. 2016. Floristic inventory, ecological characteristics and biological spectrum of plants of Parachinar, Kurram agency, Pakistan. Pak J Bot 48 (4): 1547-1558.

Blicharska M, Mikusiński G, Godbole A, Sarnaik J. 2013. Safeguarding biodiversity and ecosystem services of sacred groves-experiences from Northern Western Ghats. Intl J Biodivers Sci Ecosyst Serv Manag 9 (4): 339-346. DOI: 10.1080/21513732.2013.835350.

Blondel J. 2006. The 'design' of Mediterranean landscapes: A millennial story of humans and ecological systems during the historic period. Hum Ecol 34 (5): 713-729. DOI: 10.1007/s10745-006-9030-4

Chase MW, Christenhusz MJM, Fay MF, Byng JW, Judd WS, Soltis DE et al. 2016. An update of the Angiosperm Phylogeny Group classification for the orders and families of flowering plants: APG IV. Bot J Linn Soc 181: 1-20. DOI: 10.1111/boj.12385.

Cowling SA, Field CB. 2003. Environmental control of leaf area production: Implications for vegetation and land-surface modeling. Glob Biogeochem Cycles $17 \quad$ (1): 7-1. DOI: $10.1029 / 2002$ GB001915.

Davidson AM, Jennions M, Nicotra AB. 2011. Do invasive species show higher phenotypic plasticity than native species and, if so, is it adaptive? A meta-analysis. Ecol Lett 14 (4): 419-431. DOI: 10.1111/j.1461-0248.2011.01596.x.

de Paula LFA, Mota NFO, Viana PL, Stehmann JR. 2017. Floristic and ecological characterization of habitat types on an inselberg in Minas Gerais, Southeastern Brazil. Acta Botanica Brasilica 31 (2): 199-211. DOI: 10.1590/0102-33062016abb0409.

Farooq M, Anjum W, Khan KR, Shah AH, Shah GM, Hussain M et al. 2019. Pioneer community level syntaxonomy of forests of lesser Himalayan belt of upper Tanawal Mansehra, Pakistan. Proc Intl Acad Ecol Environ Sci 9 (4): 127-136.

Gastauer M, Neto JAAM. 2017. Updated angiosperm family tree for analyzing phylogenetic diversity and community structure. Acta $\begin{array}{lll}\text { Botanica } & \text { Brasilica } 31 & \text { (2): 191-198. DOI: 10.1590/0102- }\end{array}$ $33062016 a b b 0306$. 
Ghildiyal JC, Barthwal N, Khantwal A. 2016. Life-forms and biological spectrum of Deeva Ka Daanda a sacred grove in Garhwal Himalaya. Indian For 142 (2): 167-178.

Gnanasekaran G, Nehru P, Narasimhan D. 2012. Angiosperms of Sendirakillai Sacred Grove (SSG), Cuddalore District, Tamil Nadu, India. Check List 8 (1): 113-129. DOI:10.15560/8.1.113.

Hailemariam M. 2019. Biodiversity storehouses and showcases of sacred natural sites for nature conservation and climate change mitigation. Am J Life Sci 7 (2): 38-46. DOI 10.11648/j.ajls.20190702.11.

Haq SM, Hamid M, Lone FA, Singh B. 2021. Himalayan hotspot with alien weeds: A case study of biological spectrum, phenology, and diversity of weedy plants of high altitude mountains in District Kupwara of J \& K Himalaya, India. Proc Natl Acad Sci India Sect B Biol Sci 91 (1): 139-152. DOI: 10.1007/s40011-020-01219-6.

Haq SM, Malik AH, Khuroo AA, Rashid I. 2019. Floristic composition and biological spectrum of Keran - a remote valley of Northwestern Himalaya. Acta Ecologica Sinica 39 (5): 372-379. DOI 10.1016/j.chnaes.2018.12.001.

Hasnat GNT, Hossain MK. 2020. Global overview of tropical dry forests In: Bhadouria R, Tripathi S, Srivastava P, Singh P (eds.). Handbook of Research on the Conservation and Restoration of Tropical Dry Forests. IGI Global, USA. DOI: 10.4018/978-1-7998-0014-9.ch001.

Hunter J, Franklin S, Luxton S, Loidi J. 2021. Terrestrial biomes: A conceptual review. Veg Classif Surv 2: 73-85. DOI 10.3897/VCS/2021/61463.

International Union for Conservation of Nature (IUCN). 2021. The IUCN Red List of Threatened Species. Version 2021-1. Retrieved from https://www.iucnredlist.org, accessed on 18 September, 2021.

Kar PK, Biswal AK, Barik KL. 2010. Floristic composition and biological spectrum of a grassland community of Rangamatia in the district of Mayurbhanj, Odisha. J Curr Sci 15 (2): 465-469.

Khan W, Khan SM, Ahmad H, Alqarawi AA, Shah GM, Hussain M, Abd Allah EF. 2018. Life forms, leaf size spectra, regeneration capacity and diversity of plant species grown in the Thandiani forests, District Abbottabad, Khyber Pakhtunkhwa, Pakistan. Saudi J Biol Sci 25 (1): 94-100. DOI: 10.1016/j.sjbs.2016.11.009.

Martin PH, Canham CD, Marks PL. 2009. Why forests appear resistant to exotic plant invasions: Intentional introductions, stand dynamics, and the role of shade tolerance. Front Ecol Environ 7 (3): 142-149. DOI $10.1890 / 070096$

Mueller-Dombois D, Ellenberg H. 1974. Aims and Methods of Vegetation Ecology. Wiley and Sons, Canada.

Nafeesa Z, Haq SM, Bashir F, Gaus G, Mazher M, Anjum M et al. 2021 Observations on the floristic, life-form, leaf-size spectra and habitat diversity of vegetation in the Bhimber hills of Kashmir Himalayas. Acta Ecologica Sinica 41 (3): 228-234. DOI: 10.1016/j.chnaes.2021.03.003.

National Biodiversity Authority (NBA). 2021. Biodiversity Heritage Site. Retrieved from http://nbaindia.org/content/106/29/1/bhs.html, accessed on 18.11.2021

Nazir A, Malik ZH. 2006. Life-form and index of similarity of plan communities recorded at Sarsawa Hills, District Kotli. J Res Sci 17 (1): 27-33.

Nicotra AB, Leigh A, Boyce CK, Jones CS, Niklas KJ, Royer DL, Tsukaya H. 2011. The evolution and functional significance of leaf shape in the angiosperms. Funct Plant Biol 38 (7): 535-552. DOI: 10.1071/FP11057.

Paria N. 2005. Medicinal Plant Resources of Southwest Bengal. Vol.-1. Research Wing, Directorate of Forests in Collaboration with Department of Environment. Government of West Bengal, Kolkata, India.

Parthasarathy N, Babu KN. 2021. Sacred groves: Potential for biodiversity and bioresource management. In: Leal Filho W, Azul AM, Brandli L, Lange Salvia A, Wall T (eds.). Life on Land. Encyclopedia of the UN
Sustainable Development Goals. Springer, Switzerland. DOI: 10.1007/978-3-319-95981_10.

Pérez-Luque AJ, Bonet FJ, Pérez-Pérez R, Aspizua R, Lorite J, Zamora R. 2014. Sinfonevada: Dataset of floristic diversity in Sierra Nevada forests (SE Spain). Phyto Keys 35: 1-15. DOI: $10.3897 /$ Phytokeys.35.6363.

Prain D. 1903. Bengal Plants Vol. 1 \& 2. Botanical Survey of India, Kolkata, India.

Raunkiaer C. 1934. The Life Forms of Plants and Statistical Plant Geography. Clarendon Press, Oxford.

Reddy CS, Bagyanarayana G, Reddy KN, Raju VS. 2008. Invasive Alien Flora of India. National Biological Information Infrastructure. US Geological Survey, USA.

Reich PB, Wright IJ, Cavender-Bares J, Craine JM, Oleksyn J, Westoby M, Walters MB. 2003. The evolution of plant functional variation: Traits, spectra, and strategies. Intl J Plant Sci 164 (S3): S143-S164. DOI: $10.1086 / 374368$.

Sen UK, Bhakat RK. 2019. Assessment of the floristic composition, biological spectrum, leaf size spectra and traditional conservation management of a sacred grove in West Midnapore District, West Bengal, India. Indian For 145 (2): 156-171. DOI: $10.36808 / \mathrm{if} / 2019 / \mathrm{v} 145 \mathrm{i} 2 / 144284$.

Sen UK, Bhakat RK. 2020. Quantitative analysis of floristic composition, biological spectrum and leaf spectrum of a sacred grove in Jhargram District, West Bengal, India. Biogenesis: J Ilm Biol 8 (2): 157-171. DOI: $10.24252 /$ bio.v8i2.16407.

Sen UK, Bhakat RK. 2021a. Floristic composition and biological spectrum of a sacred grove in West Midnapore District, West Bengal, India. Acta Ecologica Sinica 41 (2): 106-119. DOI: 10.1016/j.chnaes.2020.09.005.

Sen UK, Bhakat RK. 2021b. Quantitative evaluation of biological spectrumand phenological pattern of vegetation of a sacred grove of West Midnapore District, Eastern India. Asian J For 5: 83-100. DOI: 10.13057/asianjfor/r050206.

Sharma J, Raina AK. 2017. Life-form classification and biological spectrum of Nandini wildlife sanctuary, J \& K, India. Environ Conserv J 18 (1/2): 231-237. DOI: 10.36953/ECJ.2017.181231.

Sher Z, Khan ZU. 2007. Floristic composition, life form and leaf spectrum of the vegetation of Chagharzai valley, District Buner. Pak J Plant Sci 13 (1): $57-66$

Sherwani N. 2019. Flora, life-forms and biological spectrum of Muscat Governorate. Sultan Qaboos Univ J Sci 24 (2): 95-108. DOI: $10.24200 /$ squjs.vol24iss2pp95-108.

Singh M, Kaptchuk TJ, Henrich J. 2021. Small gods, rituals, and cooperation: The Mentawai water spirit Sikameinan. Evol Hum Behav 42 (1): 61-72. DOI: 10.1016/j.evolhumbehav.2020.07.008.

West Bengal Biodiversity Board (WBBB). 2021. Biodiversity Heritage Site (BHS). Retrieved from http://wbbb.wb.gov.in/bhs.php, accessed on 18.11.2021

Westoby M, Falster DS, Moles AT, Vesk PA, Wright IJ. 2002. Plant ecological strategies: Some leading dimensions of variation between species. Ann Rev Ecol Syst 33 (1): 125-159. DOI: 10.1146/annurev.ecolsys.33.010802.150452.

World Checklist of Vascular Plants (WCVP). 2021. World Checklist of Vascular Plants, version 2.0. Facilitated by the Royal Botanic Gardens, Kew. Retrieved from http://wcvp.science.kew.org/, accessed on 18 September, 2021.

Wright IJ, Dong N, Maire V, Prentice IC, Westoby M, Díaz S et al. 2017. Global climatic drivers of leaf size. Science 357 (6354): 917-921. DOI: $10.1126 /$ science.aal4760.

Zemmar N, Bouzina MMH, Ababou A, Hedidi D. 2020. Analysis of the floristic diversity in a southern Mediterranean ecosystem. Case of Bissa forest, Chlef (Algeria). Bot Complut 44: 19-28. DOI: 10.5209/bocm.64447. 